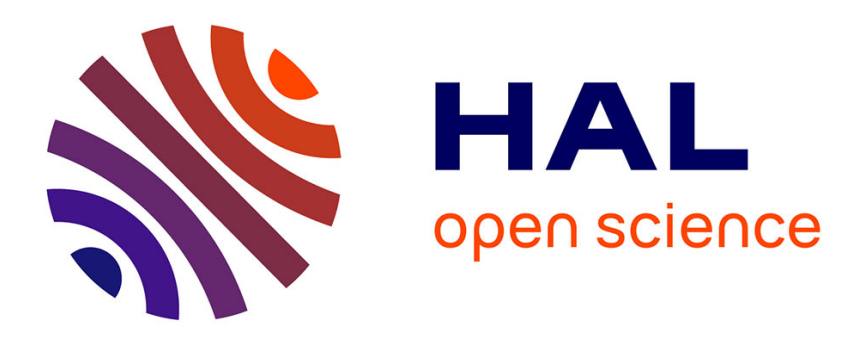

\title{
Metastable states of a spin glass chain at 0 temperature
}

B. Derrida, E. Gardner

\section{To cite this version:}

B. Derrida, E. Gardner. Metastable states of a spin glass chain at 0 temperature. Journal de Physique, 1986, 47 (6), pp.959-965. 10.1051/jphys:01986004706095900 . jpa-00210293

\section{HAL Id: jpa-00210293 https://hal.science/jpa-00210293}

Submitted on 1 Jan 1986

HAL is a multi-disciplinary open access archive for the deposit and dissemination of scientific research documents, whether they are published or not. The documents may come from teaching and research institutions in France or abroad, or from public or private research centers.
L'archive ouverte pluridisciplinaire HAL, est destinée au dépôt et à la diffusion de documents scientifiques de niveau recherche, publiés ou non, émanant des établissements d'enseignement et de recherche français ou étrangers, des laboratoires publics ou privés. 
Classification

Physics Abstracts

05.20

\title{
Metastable states of a spin glass chain at 0 temperature
}

\author{
B. Derrida and E. Gardner $\left({ }^{+}\right)$ \\ Service de Physique Théorique, CEA-Saclay, 91191 Gif-sur-Yvette Cedex, France \\ $\left(^{+}\right)$Department of Physics, University of Edinburgh, Edinburgh EH9 3JZ, U.K. \\ (Reçu le 18 décembre 1985, accepté le 29 janvier 1986)
}

\begin{abstract}
Résumé. - Nous étudions un verre de spin d'Ising à une dimension et à température nulle. Nous calculons les moments du nombre total d'états métastables et le nombre typique d'états métastables à une aimantation donnée. Nous obtenons que pour toutes les aimantations plus petites ou égales à $m_{\max }=0,446042 \ldots$, il y a un nombre exponentiellement grand d'états métastables. Au-dessus de cette valeur $m_{\max }$, il n'y a plus d'état métastable. L'aimantation rémanente $m_{\text {rem }}$ d'une chaîne est $1 / 3$ pour une dynamique où on retourne un spin à la fois quand on commence à l'instant $t=0$ avec tous les spins alignés. Notre calcul montre que $m_{\text {rem }} n$ 'est pas donnée par l'aimantation maximum des états métastables. Nos résultats sont valables pour une distribution arbitraire des couplages, à condition qu'elle soit symétrique et continue.
\end{abstract}

\begin{abstract}
We consider an Ising spin glass chain at 0 temperature. The moments of the total number of metastable states and the typical number of metastable states at a given magnetization are calculated. We find that for all magnetizations less than or equal to $m_{\max }=0.446042 \ldots$ there is an exponentially large number of metastable states. For magnetizations larger than $m_{\max }$, there are no metastable states. The remanent magnetization $m_{\mathrm{rem}}$ is known to be $1 / 3$ for single spin flip dynamics when one starts at time $t=0$ with all the spins aligned. This shows that the remanent magnetization is not given by the metastable states of maximum magnetization. Our results are valid for a spin glass chain with an arbitrary symmetric and continuous distribution of nearest neighbour interactions.
\end{abstract}

\section{Introduction.}

It has been known for a long time that dynamical effects are very important in spin glasses [1]. It is believed that the long time behaviour of spin glasses is dominated by the presence of many metastable states. However in most cases one does not know how to describe these metastable states quantitatively. The simplest questions one can ask about metastable states are : how many are they ? [2] What is the size of their basin of attraction? If one lets a spin configuration evolve in time, into what metastable state will it fall ? [3] Such questions arise both in the theory of spin glasses and in problems of pattern recognition like the Hopfield model [4].

The purpose of the present paper is to show that all these questions can be answered for the one dimensional spin glass chain at 0 temperature. First, the moments of the number of metastable states of a spin glass chain will be calculated. We shall see that the typical number of metastable states of a chain of $L$ spins is $2^{L / 3}$ (a result already obtained by $\mathrm{Li}[5]$ ) whereas the average number grows like $(4 / \pi)^{L}$. The typical number of metastable states at a given magnetization will then be calculated. We shall see that there exists a maximal magnetization $m_{\max }=0,446042 \ldots$ above which there is no metastable state. This maximal value $m_{\max }$ is significantly higher than the remanent magnetization $m_{\mathrm{rem}}=1 / 3$ (Fernandez-Medina) [6] obtained with single spin flip dynamics when one starts at time $t=0$ with magnetization 1.

\section{Number of metastable states and their moments.}

In this section the moments of the number of metastable states of an Ising spin glass chain at 0 temperature will be computed. We will see in particular that the typical number of metastable states is not equal to its average. Our results are independent of the details of the distribution $\rho\left(J_{i, i+1}\right)$ of the interactions $J_{i, i+1}$. The only condition for our results to hold is that the distribution $\rho$ is symmetric $\left(\rho\left(J_{i j}\right)=\rho\left(-J_{i j}\right)\right)$ and does not contain any delta function. So our results will be valid for a Gaussian distribution of bonds 
$\left(\rho\left(J_{i j}\right)=(2 \pi)^{-1 / 2} J^{-1} \exp \left(-J_{i j}^{2} / 2 J^{2}\right)\right)$ or for a flat distribution $\left(\rho\left(J_{i j}\right)=\theta\left(\frac{1}{2}-\left|J_{i j}\right| / J\right)\right)$.

Let us consider a chain of $L$ spins with free boundary conditions. This chain has $L-1$ bonds. For a given sample, i.e. for a given choice of the $J_{i, i+1}$ for $1 \leqslant i \leqslant$ $L-1$, the number of metastable states is always given by $2^{\omega}$ where $\omega$ is the number of weak bonds (Ettelaie and Moore) [5].

To understand this let us recall the idea used by Ettelaie and Moore. There are three kinds of bonds along the chain : strong bonds, medium bonds and weak bonds. (In order to include the bonds $J_{1,2}$ and $J_{L-1, L}$ in the discussion, we use the fact that we have chosen free boundary conditions.)

We say that $J_{i, i+1}$ is a strong bond if $\left[\left|J_{i, i+1}\right|>\right.$ $\left.\max \left(\left|J_{i-1, i}\right|,\left|J_{i+1, i+2}\right|\right)\right]$. So a bond is strong if its two neighbouring bonds are weaker. Similarly a bond $J_{i, i+1}$ is weak if $\left[\left|J_{i, i+1}\right|<\min \left(\left|J_{i-1, i}\right|\right.\right.$, $\left.\left.\left|J_{i+1, i+2}\right|\right)\right]$. So a weak bond is by definition surrounded by two stronger bonds. Lastly a bond is a medium bond if one of its neighbours is stronger and the other neighbour is weaker. With free boundary conditions it is clear that $J_{1,2}$ and $J_{L-1, L}$ are never weak.

By definition, a metastable configuration of the spins $\sigma_{i}$ is a configuration where each spin $\sigma_{i}$ is aligned in its local field. So

$$
\sigma_{i}=\operatorname{sgn}\left[J_{i-1, i} \sigma_{i-1}+J_{i, i+1} \sigma_{i+1}\right] .
$$

So in a metastable configuration each spin satisfies the stronger of the two bonds $J_{i-1, i}$ and $J_{i, i+1}$. Therefore in a metastable configuration, only weak bonds can be frustrated. If there are $\omega$ weak bonds on a chain of length $L$, then each weak bond can be either frustrated or not. Therefore the number of metastable states is just $2^{\omega}$ (we fix the spin $\sigma_{1}=+1$ ).

Let us compute the probability that a chain of $L$ spins has $\omega$ weak bonds. To do so, we shall use a recursion relation between this probability for length $L$ and for length $L+1$. Let us define $P_{L}(\omega, x)$ as the probability that a chain of $L$ spins has $\omega$ weak bonds, with the conditions that $\left|J_{L-1, L}\right|=x$ and that $\left|J_{L-2, L-1}\right|>x$. Similarly we define $Q_{L}(\omega, x)$ as the probability that the chain has $\omega$ weak bonds with the conditions that $\left|J_{L-1, L}\right|=x$ and that $\left|J_{L-2, L-1}\right|<x$. One then sees that

$$
\begin{aligned}
& P_{L+1}(\omega, x)=[\rho(x)+\rho(-x)] \times \\
& \times {\left[\int_{x}^{\infty} P_{L}\left(\omega, x^{\prime}\right)+Q_{L}\left(\omega, x^{\prime}\right) \mathrm{d} x^{\prime}\right] } \\
& Q_{L+1}(\omega, x)=[\rho(x)+\rho(-x)] \times \\
& \times\left[\int_{0}^{x} P_{L}\left(\omega-1, x^{\prime}\right)+Q_{L}\left(\omega, x^{\prime}\right) \mathrm{d} x^{\prime}\right] .
\end{aligned}
$$

These recursion relations are easy to understand.
In almost all situations, the number of weak bonds $\omega$ remains unchanged when one adds the bond $J_{L, L+1}$. The only case where $\omega$ increases by one is $\left|J_{L-1, L}\right|<$ $<\min \left(\left|J_{L-2, L-1}\right|,\left|J_{L, L+1}\right|\right)$ which corresponds to the first term in equation (2b).

It is convenient to introduce the following functions

$$
\begin{aligned}
& A_{L}(\lambda, x)=\sum_{\omega=0}^{\infty} \mathrm{e}^{\lambda \omega} P_{L}(\omega, x) \\
& B_{L}(\lambda, x)=\sum_{\omega=0}^{\infty} \mathrm{e}^{\lambda \omega} Q_{L}(\omega, x)
\end{aligned}
$$

and to introduce $\psi(x)$ defined by

$$
\psi(x)=\rho(x)+\rho(-x)=2 \rho(x)
$$

because $\rho\left(J_{i j}\right)$ is symmetric. One has of course $\int_{0}^{\infty} \psi(x) \mathrm{d} x=1$. The equations (2) become then

$$
\begin{aligned}
& A_{L+1}(\lambda, x)=\psi(x) \int_{x}^{\infty} A_{L}\left(\lambda, x^{\prime}\right)+B_{L}\left(\lambda, x^{\prime}\right) \mathrm{d} x^{\prime} \\
& B_{L+1}(\lambda, x)=\psi(x) \int_{0}^{x} \mathrm{e}^{\lambda} A_{L}\left(\lambda, x^{\prime}\right)+B_{L}\left(\lambda, x^{\prime}\right) \mathrm{d} x^{\prime}
\end{aligned}
$$

We see that the $A_{L+1}$ and $B_{L+1}$ are related to the $A_{L}$ and $B_{L}$ by a linear operator. In the limit $L \rightarrow \infty$, if we call $\mu(\lambda)$ the largest eigenvalue of this operator, one expects that $A_{L}(\lambda, x)$ and $B_{L}(\lambda, x)$ have the following behaviour

$$
\begin{aligned}
& A_{L}(\lambda, x) \sim[\mu(\lambda)]^{L} a(\lambda, x), \\
& B_{L}(\lambda, x) \sim[\mu(\lambda)]^{L} b(\lambda, x) .
\end{aligned}
$$

To find the eigenvalue $\mu(\lambda)$, one has to solve the following equations

$$
\begin{aligned}
& \mu(\lambda) a(\lambda, x)=\psi(x) \int_{x}^{\infty} a\left(\lambda, x^{\prime}\right)+b\left(\lambda, x^{\prime}\right) \mathrm{d} x^{\prime} \\
& \mu(\lambda) b(\lambda, x)=\psi(x) \int_{0}^{x} \mathrm{e}^{\lambda} a\left(\lambda, x^{\prime}\right)+b\left(\lambda, x^{\prime}\right) \mathrm{d} x^{\prime} .
\end{aligned}
$$

If we differentiate once with respect to $x$, then

$$
\begin{aligned}
& \mu a^{\prime}=\frac{\psi^{\prime}}{\psi} \mu a-(a+b) \psi \\
& \mu b^{\prime}=\frac{\psi^{\prime}}{\psi} \mu b+\left(\mathrm{e}^{\lambda} a+b\right) \psi .
\end{aligned}
$$

The equations (7) also imply the following boundary conditions

$$
\begin{aligned}
a(\lambda, \infty) & =0 \\
b(\lambda, 0) & =0 .
\end{aligned}
$$


The $\psi(x)$ dependence of the solution can be determined easily. We look for a solution of (8) which has the following form

$$
\begin{aligned}
& a(\lambda, x)=\psi(x) F(\lambda, h(x)) \\
& b(\lambda, x)=\psi(x) G(\lambda, h(x))
\end{aligned}
$$

where $h(x)$ is defined by

$$
h(x)=\int_{0}^{x} \psi(y) d y .
$$

The equations (8) become

$$
\begin{aligned}
& \mu \frac{\mathrm{d} F(\lambda, z)}{\mathrm{d} z}=-[F(\lambda, z)+G(\lambda, z)] \\
& \mu \frac{\mathrm{d} G(\lambda, z)}{\mathrm{d} z}=\mathrm{e}^{\lambda} F(\lambda, z)+G(\lambda, z)
\end{aligned}
$$

and the boundary condition (9) is replaced by

$$
\begin{gathered}
F(\lambda, 1)=0 \\
G(\lambda, 0)=0=\mu \frac{\mathrm{d} F}{\mathrm{~d} z}(\lambda, 0)+F(\lambda, 0)
\end{gathered}
$$

From (12), one can eliminate $G(\lambda, z)$ and one gets

$$
-\mu^{2} \frac{\mathrm{d}^{2} F(\lambda, z)}{\mathrm{d} z^{2}}=\left(\mathrm{e}^{\lambda}-1\right) F(\lambda, z) .
$$

The solution of (14) which satisfies (13a) is

$$
F(\lambda, z)=\sin \left[\frac{\sqrt{\mathrm{e}^{\lambda}-1}}{\mu}(1-z)\right]
$$

and the condition (13b) fixes the value of $\mu$

$$
-\mu \frac{\sqrt{\mathrm{e}^{\lambda}-1}}{\mu} \cos \left(\frac{\sqrt{\mathrm{e}^{\lambda}-1}}{\mu}\right)+\sin \left(\frac{\sqrt{\mathrm{e}^{\lambda}-1}}{\mu}\right)=0
$$

i.e.

$$
\mu=\sqrt{\mathrm{e}^{\lambda}-1} / \operatorname{Arctang}\left(\sqrt{\mathrm{e}^{\lambda}-1}\right) .
$$

So we see that for large $L$

$$
\lim _{L \rightarrow \infty} \frac{1}{L} \log \left\langle\mathrm{e}^{\lambda \omega}\right\rangle=\log (\mu(\lambda))
$$

where $\mu(\lambda)$ is given by (17).

Let us now come back to the number $N$ of metastable states of a chain of length $L$. We have already seen that $N=2^{\omega}$. Therefore we can compute all the moments of $N$.

$$
\left\langle N^{p}\right\rangle=\left\langle\mathrm{e}^{\omega p \log 2}\right\rangle
$$

and one finds

$$
\begin{aligned}
\lim _{L \rightarrow \infty} \frac{1}{L} \log \left(\left\langle N^{p}\right\rangle\right) & =\log (\mu(p \log 2))= \\
= & \log \left[\frac{\sqrt{2^{p}-1}}{\operatorname{Arctang}\left(\sqrt{\left.2^{p}-1\right)}\right.}\right] .
\end{aligned}
$$

Notice that $p$ needs not be an integer. From this we can compute for example $\langle\log N\rangle$ in agreement with the result of $\mathrm{Li}$ [5]

$$
\begin{aligned}
\lim _{L \rightarrow \infty} \frac{1}{L}\langle\log N\rangle & =\lim _{p \rightarrow 0} \frac{\log (\mu(p \log 2))}{p} \\
& =\frac{\log 2}{3}=0.2310 \ldots
\end{aligned}
$$

Similarly one can compute the average number $\langle N\rangle$ of metastable states

$$
\lim _{L \rightarrow \infty} \frac{1}{L} \log \langle N\rangle=\log \left(\frac{4}{\pi}\right) \simeq 0.2416 \ldots
$$

So we see that the typical number $\mathrm{e}^{\langle\log N\rangle}$ of the metastable configurations is not equal to the average number $\langle N\rangle$ of metastable states. The same thing is probably true for all spin glass models in finite dimension but for the S. K. model [2] Bray and Moore have argued that $\log \langle N\rangle=\langle\log N\rangle$.

\section{Typical number of metastable states at magnetiza- tion $m$.}

As we discussed above, for each sample the number of metastable states is equal to $2^{\omega}$ where $\omega$ is the number of weak bonds. A metastable state always has all its medium and strong bonds unfrustrated whereas the weak bonds can be either frustrated or unfrustrated. So the chain is broken into clusters of medium and strong bonds, each cluster being delimited by two consecutive weak bonds. Since the weak bonds are either frustrated or unfrustrated, two clusters separated by a weak bond are independent.

In this section, we will first give the expression of the typical number $L X_{l}$ of clusters of $l$ spins between two consecutive weak bonds for a chain of length $L$. From the knowledge of the $X_{l}$, we shall compute the typical number of metastable states at a given magnetization $m$ per spin.

In the appendix, we show that

$$
X_{l}=\frac{2^{l}(l-1)(l+2)}{(l+3) !} .
$$

Each cluster of $l$ spins is independent of its neighbours and all the $l-1$ bonds in this cluster are satisfied. So all the spins of a cluster are rigid. Because the distribution of bonds is symmetric, the probability $P_{l, n}$ that a cluster of $l$ spins has a magnetization whose.modulus 
is $n$ is given by

$$
\begin{aligned}
& P_{l, n}=\frac{1}{2^{l}}\left[{C_{l}^{\frac{l+n}{2}}}^{\frac{l-n}{2}}\right]=\frac{2 l !}{2^{l}\left(\frac{l+n}{2}\right) !\left(\frac{l-n}{2}\right) !} \\
& \text { for } n \geqslant 1 \text { (24a) } \\
& P_{l, 0}=\frac{1}{2^{l}} \frac{l !}{\left[\left(\frac{l}{2}\right) !\right]^{2}} \text { for } n=0
\end{aligned}
$$

These formulae should only be used for $n-l$ even. Therefore the typical number $L Y_{l, n}$ of clusters of $l$ spins and of magnetization $|n|$ is given by

$$
Y_{l, n}=2 \frac{(l-1)}{(l+3)(l+1)} \frac{1}{\left(\frac{l+n}{2}\right) !\left(\frac{l-n}{2}\right) !}
$$

for $n \geqslant 1, l-n \geqslant 0, l-n$ even

$$
Y_{l, 0}=\frac{l-1}{(l+3)(l+1)} \frac{1}{\left[\left(\frac{l}{2}\right) !\right]^{2}}
$$

for $n=0, l$ even and larger than 2 .

By summing over $l$, one can deduce that the typical number $L Z_{n}$ of clusters of magnetization $n$

$$
\begin{aligned}
& Z_{n}=\sum_{i=0}^{\infty} \frac{2(2 i+n-1)}{(2 i+n+3)(2 i+n+1)} \frac{1}{(i+n) ! i !} \\
& Z_{0}=\sum_{i=1}^{\infty} \frac{2 i-1}{(2 i+3)(2 i+1)} \frac{1}{(i !)^{2}} .
\end{aligned}
$$

From the knowledge of the $Z_{n}$, it is easy to compute the maximal magnetization $\mathrm{Lm}_{\max }$ of metastable states of a chain of $L$ sites. $m_{\max }$ is obtained by requiring that all the clusters which have a magnetization $n$ point in the same direction. Therefore

$$
m_{\max }=\sum_{n=0}^{\infty} n Z_{n}
$$

Similarly one can compute the typical number of metastable states at magnetization $\mathrm{Lm}$. To do so, let us consider that when the magnetization is $L m$, there are $\frac{1+\alpha_{n}}{2} L Z_{n}$ clusters having a magnetization $+n$ and $\frac{1-\alpha_{n}}{2} L Z_{n}$ clusters having a magnetization $-n$. The $\alpha_{n}$ must satisfy

$$
\sum_{n=0}^{\infty} n \alpha_{n} Z_{n}=m
$$

For each choice of the $\alpha_{n}$, the number $\Omega\left(\left\{\alpha_{n}\right\}\right)$ of possible arrangements is given by

$$
\Omega\left(\left\{\alpha_{n}\right\}\right)=\prod_{n=0}^{\infty} \frac{\left(L Z_{n}\right) !}{\left.L Z_{n}\left(\frac{1+\alpha_{n}}{2}\right)\right] !\left[L Z_{n}\left(\frac{1-\alpha_{n}}{2}\right)\right] !}
$$

The number $N(m)$ of metastable configurations having a magnetization $\mathrm{Lm}$ is then simply given by

$$
\langle\log N(m)\rangle=\max _{\left\{\alpha_{n}\right\}} \log \Omega\left(\left\{\alpha_{n}\right\}\right)
$$

with the constraint (28) on the $\alpha_{n}$. One has of course

$$
\begin{aligned}
& \log \Omega\left(\left\{\alpha_{n}\right\}\right)=L \sum_{n=0}^{\infty}-Z_{n} \times \\
& \times\left[\frac{1+\alpha_{n}}{2} \log \left(\frac{1+\alpha_{n}}{2}\right)+\frac{1-\alpha_{n}}{2} \log \left(\frac{1-\alpha_{n}}{2}\right)\right] .
\end{aligned}
$$

Using a Lagrange multiplier $\beta$, one finds that the $\alpha_{n}$ have the form

$$
\alpha_{n}=\tanh (\beta n)
$$

and

$$
\begin{aligned}
\lim _{L \rightarrow \infty} \frac{1}{L}\langle\log N(m)\rangle=\sum_{n=0}^{\infty} Z_{n} \times \\
\times[\log (2 \cosh \beta n)-\beta n \tanh (\beta n)]
\end{aligned}
$$

$\frac{1}{L}\langle\log N(m)\rangle$ can be drawn as a function of $m$. The curve is parametrized by $\beta, \beta$ going from $-\infty$ to $+\infty$ and $m$ is given by

$$
m=\sum_{n=0}^{\infty} n Z_{n} \tanh (\beta n) .
$$

The maximum number of metastable states are those of 0 magnetization corresponding to $\beta=0$.

$$
\langle\log N(0)\rangle=\log 2 \sum_{n=0}^{\infty} Z_{n}=\frac{1}{3} \log 2
$$

which is again the result (21) obtained by $\mathrm{Li}$ [5]. When $m$ increases, $\langle\log N(m)\rangle$ decreases until $\beta \rightarrow \infty$ where $m$ reaches the value $m_{\max }$ given by (27). For magnetizations $m>m_{\max }$, there is no metastable state with probability 1 . It is interesting to notice that $\left\langle\log N\left(m_{\max }\right)\right\rangle \neq 0$. There are an exponentially large number of metastable configurations at magnetization $m_{\max }$

$$
\lim _{L \rightarrow \infty} \frac{1}{L}\left\langle\log N\left(m_{\max }\right)\right\rangle=Z_{0} \log 2 .
$$

The reason is easy to understand. There are a macroscopic number $L Z_{0}$ of clusters with zero magnetization. These clusters can be flipped without changing the total magnetization. 
Equations (32) and (33) can be used to determine $\langle\log N(m)\rangle$ numerically. The results are plotted in figure 1 . One finds that

$$
\begin{gathered}
m_{\max }=0.446042 \ldots \\
\lim _{L \rightarrow \infty} \frac{1}{L}\left\langle\log N\left(m_{\max }\right)\right\rangle=0.06268 \ldots
\end{gathered}
$$

\section{Remanent magnetization of a spin glass chain at 0 temperature and basin of attraction of metastable states.}

In this section we address the following question : if at time $t=0$, one starts with a completely polarized configuration (all spins $\sigma_{i}=+1$ at time $t=0$ ), what will be the remanent magnetization at time $t=\infty$. The answer to that question obviously depends on the dynamics of the spins. The dynamics we want to discuss is the most natural one. At each time step, a spin $\sigma_{i}$ is chosen at random. If this spin is aligned with its local field, then it remains unchanged. Otherwise it is flipped. This procedure is iterated until the system falls into a metastable state

$$
\sigma_{i}^{t+1}=\operatorname{sgn}\left[J_{i, i+1} \sigma_{i+1}^{t}+J_{i, i-1} \sigma_{i-1}^{t}\right] .
$$

An interesting question is to know what will be the remanent magnetization $m_{\text {rem }}$ of the metastable state into which the system will fall. The answer to this question is

$$
m_{\mathrm{rem}}=\frac{1}{3}
$$

and the answer is the same for any distribution of the bonds which is symmetric and continuous.

Result (39) was first obtained by Fernandez and Medina [6]. Let us explain briefly here where it comes from. First the chain is composed of clusters delimited by weak bonds. Each cluster of $l$ sites has one strong bond and $l-2$ medium bonds. The total number of clusters is equal to the total number of weak bonds, i.e. is equal to $L / 3$. At time $t=0$, if we start with $m=1$, half of the clusters have their strong bond unfrustrated and half of the clusters have their strong bond frustrated.

For a cluster whose strong bond is not frustrated at time $t=0$, the two spins which are connected by this strong bond will remain + for ever. The time evolution of the cluster will stop when all the medium bonds become unfrustrated. Since the signs of the bonds are random, when one averages over all such clusters, one finds that the average remanent magnetization is 2 per cluster. It is just the contribution of the two spins which are connected by the strong bond.

For similar reasons, one can understand that the average remanent magnetization of a cluster whose strong bond is frustrated at time $t=0$ will be 0 . One of the two spins connected to the strong bond will flip and then all the other spins of the cluster will adjust

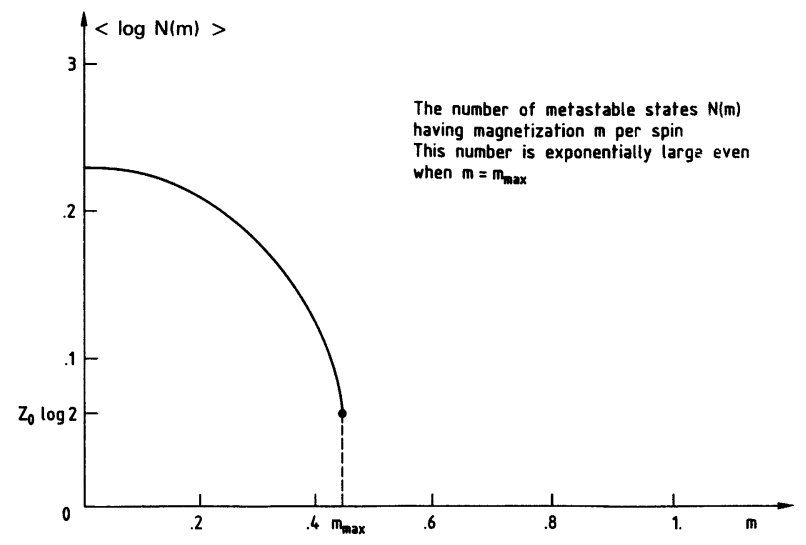

Fig. 1. - The number of metastable states $N(m)$ having magnetization $m$ per spin. This number is exponentially large even when $m=m_{\max }$.

themselves in order to satisfy all the medium bonds of the cluster.

When one adds the average remanent magnetizations of the two kinds of clusters multiplied by their numbers, one finds (39).

It is clear throughout this paper that the weak bonds do not play any role in the dynamics. Therefore if one starts at time $t=0$ with an arbitrary configuration of spins and one uses the single spin flip dynamics described above, then at $t=\infty$, each weak bond will be frustrated with probability $\frac{1}{2}$ and satisfied with probability $\frac{1}{2}$. This means that if one defines the size of the basin of attraction of a metastable state as the number of spin configurations which fall onto this metastable state, then all the metastable states have basins of attraction of the same size. (This property is probably true only in one dimension.)

\section{Discussion.}

In this paper we have seen that many properties of the metastable states of a spin glass chain can be computed exactly.

Our main result is that there is a critical value of the magnetization $m_{\max }$ above which there is no metastable state. The number of metastable states is exponentially large at all magnetizations less than or equal to this maximal magnetization $\mathbf{0 . 4 4 6 0 4 2}$. We have also seen that if the system evolves according to a single spin flip dynamics and if one starts at $t=0$ with all the spins $\sigma_{i}=+1$, then the remanent magnetization at $t=\infty$ is $1 / 3$. So the magnetization decreases from 1 to $1 / 3$ with time. It is interesting to notice that the system does not stop at the magnetization $m_{\max }=0.446042 \ldots$ So the remanent magnetization is not given by the maximal magnetization of metastable states. In other words, when one starts with magnetization $m=1$ at $t=0$, the system does 
not fall into the closest metastable states whose magnetization is $m_{\max }$.

We believe that all the results discussed in this conclusion should remain qualitatively the same for spin glass and pattern recognition models in higher dimensions.

\section{Appendix.}

In this appendix we derive formula (23) which gives the number $L X_{l}$ of clusters of $l$ sites delimited by two weak bonds in a chain of $L$ spins. To do so, we again use a transfer matrix technique.

Assume that we have a chain of $L$ spins. Let us call $c_{L}(x, l)$ the probability that the last weak bond was the bond $J_{L-l, L-l+1}$, that $\left|J_{L-1, L}\right|=x$ and that $\left|J_{L-2, L-1}\right|>x$. Similarly $d_{L}(x, l)$ is the probability that the last weak bond is $J_{L-l, L-l+1}$, that $\left|J_{L-1, L}\right|=x$ and that $\left|J_{L-2, L-1}\right|<x$.

One can write recursion relations for these probabilities

$$
\begin{aligned}
& c_{L+1}(x, l)=[\rho(x)+\rho(-x)] \times \\
& \quad \times \int_{x}^{\infty} c_{L}\left(x^{\prime}, l-1\right)+d_{L}\left(x^{\prime}, l-1\right) \mathrm{d} x^{\prime} \text { for } l \geqslant 3
\end{aligned}
$$

$$
d_{L+1}(x, 2)=[\rho(x)+\rho(-x)] \int_{0}^{x} \sum_{l=3}^{\infty} c_{L}\left(x^{\prime}, l\right) \mathrm{d} x^{\prime}
$$

$$
\begin{aligned}
d_{L+1}(x, l)= & {[\rho(x)+\rho(-x)] \times } \\
& \times \int_{0}^{x} d_{L}\left(x^{\prime}, l-1\right) \mathrm{d} x^{\prime} \quad l \geqslant 3 .
\end{aligned}
$$

It is also easy to see from the definition that $c_{L}(x, l)=0$ for $l \leqslant 2$ and $d_{L}(x, l)=0$ for $l \leqslant 1$.

In the limit $L \rightarrow \infty$, the $L$ dependence disappears and so $c_{L}(x, l) \rightarrow c(x, l)$ and $d_{L}(x, l) \rightarrow d(x, l)$. If we again introduce

$$
\psi(x)=\rho(x)+\rho(-x)
$$

then one has to solve

$$
\begin{aligned}
& c(x, l)=\psi(x) \int_{x}^{\infty} c\left(x^{\prime}, l-1\right)+d\left(x^{\prime}, l-1\right) \mathrm{d} x^{\prime} \\
& \text { for } l \geqslant 3 \\
& d(x, 2)=\psi(x) \int_{0}^{x} \sum_{l=3}^{\infty} c\left(x^{\prime}, l\right) \mathrm{d} x^{\prime} \\
& d(x, l)=\psi(x) \int_{0}^{x} d\left(x^{\prime}, l-1\right) \mathrm{d} x^{\prime} \text { for } l \geqslant 3 .
\end{aligned}
$$

As in section I of the paper, it is convenient to introduce

$$
h(x)=\int_{0}^{x} \psi(y) d y
$$

and to look for solutions which have the form

$$
\begin{aligned}
& c(x, l)=\psi(x) F(h(x), l) \\
& d(x, l)=\psi(x) G(h(x), l) .
\end{aligned}
$$

The equations for $F$ and $G$ become

$$
\begin{aligned}
& F(z, l)=\int_{z}^{1} F\left(z^{\prime}, l-1\right)+G\left(z^{\prime}, l-1\right) \mathrm{d} z^{\prime} \\
& \text { for } l \geqslant 3 \\
& G(z, 2)=\int_{0}^{z} \sum_{l=3}^{\infty} F\left(z^{\prime}, l\right) \mathrm{d} z^{\prime} \\
& G(z, l)=\int_{0}^{z} G\left(z^{\prime}, l-1\right) \mathrm{d} z^{\prime} \text { for } l \geqslant 3 .
\end{aligned}
$$

The solutions of these equations are

$$
\begin{aligned}
& F(z, l)=\frac{z^{l}}{2 l !}-\frac{z^{l-1}}{2(l-1) !}-\frac{(2-z)^{l}}{2 l !}+\frac{(2-z)^{l-1}}{2(l-1) !} \\
& \text { (A.14) } \\
& G(z, l)=\frac{z^{l-1}}{(1-1) !}-\frac{z^{l}}{l !} .
\end{aligned}
$$

Now we can compute the average number $L X_{l}$ of clusters of $l$ spins in a chain of $L$ spins.

One has

$$
X_{l-1}=\int_{0}^{\infty} \mathrm{d} x c(x, l) \int_{x}^{\infty} \psi\left(x^{\prime}\right) \mathrm{d} x^{\prime} .
$$

This equation means that if the last weak bond was $J_{L-l, L-l+1}$, if $\left|J_{L-1, L}\right|=x$, if $\left|J_{L-2, L-1}\right|>x$ and if $\left|J_{L, L+1}\right|=x^{\prime}>x$, then one has formed a cluster of $l-1$ spins by adding the bond $J_{L, L+1}$. The cluster is composed by the spins $L-l+1, L-l+2, \ldots$, $L-1$. (A.16) can be transformed into

$$
X_{l-1}=\int_{0}^{\infty} \mathrm{d} x \psi(x) F(h(x), l) \int_{x}^{\infty} \psi\left(x^{\prime}\right) \mathrm{d} x^{\prime}
$$

If we make the change of variable $z=h(x)$, then $\mathrm{d} z=\psi(x) \mathrm{d} x$ and therefore

$$
\begin{aligned}
& X_{l-1}=\int_{0}^{1} \mathrm{~d} z F(z, l)(1-z) \\
& X_{l-1}=\frac{2^{l-1}}{(l+2) !}(l-2)(l+1) .
\end{aligned}
$$

This proves formula (23). 


\section{Acknowledgments.}

Financial support for one of us (E. G.) was from the Science and Engineering Research Council, U.K.,
B. D. would like to thank Professor D. Sherrington for his hospitality at Imperial College where part of this work was done.

\section{References}

[1] Mackenzie, N. D., Young, A. P., Phys. Rev. Lett. 49 (1981) 301.

Binder, K. (Proc. Heidelberg, Colloq. on Spin Glasses Springer (83)) and references therein.

[2] Bray, A. J., Moore, M. A., J. Phys. C 14 (1981) 1313. Bray, A. J., Moore, M. A., J. Phys. C 13 (1980) L469.

Tanaka, F., Edwards, S. F., J. Phys. F 10 (1980) 2769.

De Dominicis, C. Gabay, M., Garel, T., ORland, H., J. Physique 41 (1980) 933.

[3] Kinzel, W., Preprint 1985.

Parga, N., Parisi, G., Preprint 1985.
WeisBuch, G., J. Physique-Lett. 46 (1985) L-623.

[4] Hopfield, J. J., Proc. Nat. Acad. Sci. USA 79 (1982) 2554.

Amit, D. J., Gutfreund, H., Sompolinsky, H., Phys. Rev. A 32 (1985) 1007.

Amit, D. J., Gutrreund, H., Sompolinsky, H., Phys. Rev. Lett. 55 (1985) 1539.

[5] Ettelaie and Moore, M. A., J. Physique-Lett. 46 (1985) L-893.

LI, T., Phys. Rev. B 24 (1981) 6579.

[6] Fernandez, Medina, Phys. Rev. B 19 (1979) 3561. 